\title{
Strengths and weaknesses of traditional Chinese medicine and Western medicine in the eyes of some Hong Kong Chinese
}

\author{
T P Lam
}

\begin{abstract}
Objective-To explore the attitudes of Hong Kong Chinese towards the strengths and weaknesses of traditional Chinese medicine (TCM) and Western medicine.

Design-Qualitative study of subjects' opinions using semi-structured focus group interviews.

Setting-Southern district of Hong Kong Island where many of the residents have a fisherman background.

Participants-Twenty nine participants took part in eight focus group interviews. Measurements and main resultsParticipants' attitudes towards TCM and Western medicine were explored in the interviews. Both TCM and Western medicine are used concurrently by many people in Hong Kong. Patients make decisions on which type of doctors they want to see for the specific illnesses that they are suffering from. They consider both types of medicines to have strengths and weaknesses: TCM being better in curing the root of the problem but quite slow in action while Western medicine is more powerful but sometimes too powerful with significant side effects.

Conclusions-It is important for medical practitioners to be aware of the health attitudes of their patients from different ethnic backgrounds. It will lead to a better patient-doctor relationship and better compliance of treatment.

(F Epidemiol Community Health 2001;55:762-765)
\end{abstract}

Complementary medicine is gaining popularity in the Western world. ${ }^{1}$ In other societies, for example, China and India, traditional medical practices have been widely used by the local people for hundreds of years. The trend of international migration in recent decades means that medical practitioners in the Western countries are quite likely to see patients of different ethnic origins, who are also quite likely to be using traditional therapies of their countries of origin. ${ }^{2}$

Despite the dominance of Western medicine in Hong Kong, which is considered a result of the favoured position enjoyed by Western medicine during the British rule, ${ }^{3}$ both Western and Chinese medicines have been used by the local population concurrently. The majority of the population probably explains and understands their illnesses in Chinese medical concepts rather than Western medical principles. ${ }^{4}$ For example, weakness may be explained as lack of "huet hei" (blood and energy).

Food as a form of folk therapy within the Chinese culture has also attracted some research interest. ${ }^{67}$ Koo pointed out that proper selection, timing and preparation of food were the most salient lay methods of dealing with the prevention and treatment of many common symptoms and illnesses by the local Chinese in Hong Kong. ${ }^{8}$ The food prescriptions and proscriptions are based on the traditional concept of maintaining the body homoeostasis.

It is well known that not every ill person consults a health care professional. Lau explored this issue for Hong Kong Chinese patients. He pointed out that like most patients, it is often some "triggers" that bring the patients to their healers. ${ }^{9}$ Social and cultural factors may influence the pattern of symptomatology and phenomenology. ${ }^{10}$ Literature search found little was available on how the Chinese people viewed their own traditional medical practices in comparison with Western medicine. Furthermore, most of these kinds of studies used survey questionnaires, which had the risk of collecting superficial or socially acceptable information. Qualitative methods were therefore used to collect data for this study, which was conducted to investigate the attitudes of Chinese towards the strengths and weaknesses of traditional Chinese medicine (TCM) and Western medicine in order to allow health care workers a better understanding of the health practices of their Chinese patients.

\section{Background}

TCM has been practised in China for over 2000 years. Both Western medicine and TCM are widely available in Hong Kong. Before the return of sovereignty to China in 1997, the British Hong Kong Government adopted a non-interventionist approach towards the practice of Chinese medicine in Hong Kong. Any adults of Chinese descent were allowed to be registered to practise any form of Chinese medicines but its developments otherwise received very little government attention, and certainly no support. On the other hand, Western medicine was the only form of medicine receiving formal government recognition and support. Hong Kong Medical College, which subsequently amalgamated with the University of Hong Kong, was established to provide Western medical training in Hong Kong over 
Table 1 Characteristics of subjects interviewed in the study

\begin{tabular}{lll}
\hline & Number & $\%$ \\
\hline Age & 4 & \\
$30-39$ & 2 & 14 \\
$40-49$ & 2 & 7 \\
$50-59$ & 10 & 34 \\
$60-69$ & 7 & 24 \\
$70-79$ & 4 & 14 \\
$80-89$ & 11 & 38 \\
Sex & 18 & 62 \\
Male & 18 & \\
Female & & 17 \\
Religion & 5 & 79 \\
Christian & 23 & 3 \\
Traditional & 1 & \\
None & & \\
\hline
\end{tabular}

100 years ago. Throughout the past century, the Western medical health care system developed and flourished in Hong Kong. By 1999, there were over 9000 doctors registered in Hong Kong serving a population of almost 7 million. Most of the hospital care is provided in the public hospital system. On the other hand, over $80 \%$ of the primary care is provided by 3000 private general practitioners. The Government's general outpatient clinics and other registered practitioners, including practitioners of TCM, provide the other $20 \%$ of primary care in Hong Kong. TCM practitioners are widely available in Hong Kong, but they only need to acquire a business registration without any assessment of their qualifications or standard of training and, hence, their standard varies. (This however will be changed when a new registration system with well defined assessment criteria is to be introduced soon by the post-1997 government of the Hong Kong Special Administrative Region.) They are often attached to a herbal medicinal shop and their charges are often no more than US\$2-3. Depending on the herbs prescribed, the total costs are often less than US $\$ 7-8$ per consultation.

\section{Methods}

There are different forms of TCM such as acupuncture, herbal medicine and bone setting. For the purposes of this study, TCM refers to herbal medicine only and this is also the common practice of Hong Kong Chinese.

SUBJECTS

Subjects were recruited verbally by the author or a research assistant from a primary care clinic where the author works or a hostel for the elderly, both of which are situated in the southern side of the Hong Kong Island where many of the residents have a fisherman background. Verbal invitation was given because many of the local people, especially the elderly ones, are illiterate and they might feel intimated if a written note was given to them. Family members of the patients were also recruited to participate in the study. Purposive sampling was adopted to ensure a range of demographic variables and experiences. Only four refusals were encountered and they were mainly attributable to non-availability. The characteristics of the subjects are detailed in table 1 .
STUDY DESIGN

Eight group interviews with 29 participants were conducted between March 1997 and June 1997. Group interviews consisted of up to five participants. A trained research assistant conducted these interviews using the local language, Cantonese, at the author's office or the hostel for the elderly where some of the participants were living. She was presented to the participants as an "assistant in research" for the author and was well received. The participants were also not noted to be more inhibited at either site. Each interview lasted one to one and a half hours. The participants were encouraged to tell their story and express their opinions but a checklist was used to ensure all relevant domains were covered. These included the participants' decision on the type of doctors to attend, theirs views on the strengths and weaknesses of TCM and Western medicine.

This study was approved by the Ethics Committee of the Faculty of Medicine of the University of Hong Kong.

Translation and transcription

The entire tape recorded interviews were simultaneously translated and transcribed verbatim by the research assistant and were checked by the author who listened to the recording while checking the accuracy of the transcripts.

\section{Analysis}

Transcripts were analysed using NUD ${ }^{\star}$ IST software and a grounded theory approach. ${ }^{11}$ The complete texts of the interviews were entered onto the database and they were then coded into broad themes. The objective of the analysis was to identify provisional inferences from the texts. The software, NUD`IST, with its powerful cross linking capacities permitted consideration of all relevant texts and easy modification of the broad themes. The consistency and validity of analysis and interpretation was assessed by having four randomly selected interview transcripts independently coded by two experienced qualitative researchers. The results were compared and there were no significant inconsistencies. The interpretation of the data was also discussed with five randomly selected participants who had been interviewed to compare my perspectives with that of the participants. This process again revealed no significant corrections of my interpretation.

\section{Results}

Both TCM and Western medicine are used concurrently by many people in Hong Kong. Patients do however make decisions on which type of doctors they want to see for the specific illnesses that they are suffering from.

TCM is considered to be good for some milder illnesses, for example, coughs and colds. It is also considered to be better for "clearing" the disease. It is often used as a supplement to Western medication - that is, after consulting the Western doctor for quick recovery (see below), Chinese medication is used to "clear the root of the disease", "completely cure the disease" or "cut the tail of the illness". 
Participant A: Herb medicines are better than Western medicines. Herb medicines are good for coughs, if a person can stand it [the taste]. (M/56) Participant B: Herb medicines cure diseases slower. They are good in the sense that they "don't leave a tail". Chinese medicines are better in curing diseases completely, though they are slower. (M/62)

Sometimes, Chinese medicine is used only as an "alternative". When Western medication fails, or when you are so desperate you want to try anything, Chinese medication will be used. It is also good in dealing with the side effects of taking Western medicines, like "the bitterness in the mouth", "the sputum", and the "wind".

Participant C: After I have taken Western medicines for a long time, I will see the Chinese doctor to clear the bitterness in my mouth. I always feel that the tongue is rough, a bit bitter. After I take a bowl of Chinese medicine, I don't have that kind of feeling any more. Maybe it is good for "clearing the heat". ( $\mathrm{F} / 40)$

Chinese medicine is considered to be milder and does not have as strong side effects as Western medicine.

Interviewer: Under what conditions will you make [herbal] tea for yourself? What disease?

Participant D: When we cough, we make "gut beng" and "woo dau" for the "hot cough". Usually the young people have "hot cough". We boil "wan yee" and "woo dau" for them. If it doesn't work, we will go to see a [Western] doctor... For us [the elderly], we are afraid of "cold cough". When we have "cold cough", we boil "gut beng" "woo dau" and "geong chin". If it doesn't work, I will go to see a [Western] doctor. (F/60)

TCM is however seen to have some specific weaknesses. It is less convenient than Western medicine because you have to boil the herbs to make the medicine, and very often, you have to see the Chinese doctor many times before the disease is really cured. The herbal medicine almost always tastes very bitter, which makes it unpopular with some patients. On taking Chinese medicine, the patient has to avoid certain food, "gai hau", and this is difficult for some others. It is also considered to be slow in action. This may lead to delay of proper treatment, or worsening of the disease for example, the flu virus may go to other parts of the body, causing more serious diseases or death.

Participant E: If you have a sore hand, it takes you a really long time to cure it with Chinese medicines only. (M/75)

Participant F: Taking Chinese medicines, the disease won't be cured by one dose only. You have to take a few doses. $(\mathrm{M} / 70)$

On the other hand, Western medicine is considered to have some certain strengths. It is more convenient than TCM herbal teas because there is no need to boil the herbs. It produces faster recovery: you can even choose injections to hasten the recovery.

Participant F: If I do not get well after I have consulted the Western doctor, I will see the Chinese doctor. $(\mathrm{F} / 55)$

Interviewer: That means you would choose the Western doctor first.
Participant F: See the Western doctor first? Usually we do, because we can get well sooner. It takes time to cure the disease by Chinese medication. Chinese medication is better in its power to clear the root of the illness. Western medication is faster, but it is less powerful in clearing the illness.

Western medicine also better to cure some diseases immediately for example, flu, as accumulation of the virus is considered by some patients to lead to adverse effects on health. It is sometimes better to use Western medication to control the symptoms first, and then use Chinese medication to "completely cure the disease".

Participant C: After I have visited a Western doctor for my cold and the cold still continues for a very long time, I will go to see a Chinese doctor. Interviewer: What do you mean?

Participant C: That means when the cold cannot be "cleared" [by Western medicine].

Western medicine is included in the employee's medical insurance schemes for some workers in Hong Kong. Hence, they may pay less out of pocket. Another advantage is that there is no need to avoid certain food-that is, "gai hau". Western medicine is also considered better for certain illnesses, for example, tuberculosis, because injection is available.

Western medicine is however considered to have some distinct weaknesses. It is considered to have significant side effects, leading to "fat tseng gwong" (feeling dull), bitterness in the mouth, sputum, weakness, and the "wind".

Participant B: I think Chinese medicine is milder. After we take Western medicine, we feel dull. Chinese medicine is rarely like that. It is better. Say if you go to see Doctor X ... his medicine is good, very strong. When you have a sore throat or a cold, he can really cure your coughing immediately. However, the body feels...

Participant A: Weak. It is as if you are going to rise to the heaven.

Furthermore, Western medicine is thought to be "ba" (too powerful). Taking pills too often is therefore not good for the body.

Participant G: After a person takes a pill, the pain goes away after a short while. Isn't it that the Western medicine is "ba"? The medicine affects the body of the people very strongly. Thus the intestines are less painful. Don't say that the pills are good. If you take them too often, it is not good to the health. $(M / 70)$

\section{Discussion}

\section{THE STUDY}

This study aimed to explore a major area of health beliefs of a group of Hong Kong Chinese. Qualitative methods were used because they permitted an in depth exploration of the opinions of the people. The data obtained in this study would not have been possible if a questionnaire survey methodology were to be adopted. The fact that the author has practised in the community for five years allowed him to recruit subjects with diverse demographic background. Efforts were also made to recruit subjects from outside of the author's clinic. This had helped to ensure as wide a recruitment population as possible. Because of the 
"presumed" status of the author in the community, a trained research assistant was responsible to conduct the interviews to minimise that impact. This helped to relieve the anxiety that some of the participants would have of their personal family doctors in an unfamiliar non-clinical setting. A limitation of this study is, however, that a modest number of 29 people were interviewed and it may be possible that the opinions expressed by these participants to be slightly different from the views of some other Hong Kong Chinese people.

IMPLICATIONS FOR CLINICAL PRACTICE

This study shows the attitudes of some Hong Kong Chinese towards TCM and Western medicine. It shows that they consider both types of medicines to have strengths and weaknesses. They therefore choose TCM or Western medicine for the specific types of illness that they are suffering from.

By understanding patients' views on conventional and complementary medicine, it would encourage medical practitioners in Western countries to begin discussions with their Chinese patients on some concerns that they may have about Western medications. The fact that Chinese patients may consider Western medicine to have significant side effects and may also be too powerful may lead some of them to take "holiday" from their long term Western medications for chronic illnesses like diabetes and hypertension and, hence, affects their drug compliance.

On the other hand, the belief in the "faster" action of Western medicine may lead some Chinese patients to be over-dependent on medications and may have higher expectations in drug prescription, even for illnesses that medications are not necessary or even harmful. One clinical example that I often come across is that some Hong Kong Chinese parents expect their children suffering from vomiting attributable to self limiting causes, including gastroenteritis, to be prescribed anti-vomiting drugs that may have serious side effects, particularly in children. I have had cases where parents had given their own anti-vomiting drugs to their young children, hoping that it would stop their vomiting. This is an example why it is important for the doctors to beware of the health belief and practice of their ethnic patients. They may consider to actively provide appropriate patient education to the patients or carers.

Furthermore, some Chinese patients may be already taking TCM medications when they consult their Western doctors. This may expose them to potential interactions between TCM and Western medications with significant health effects. ${ }^{12}$ To improve the patient-doctor relationship, it might also be useful if the doctors who look after Chinese patients can use terms similar to their patients' and, hence, facilitate, communication.

This study, as well as other studies, ${ }^{13}$ has shown that patients who use complementary medicine tend to use conventional medicine concurrently. It would also improve the patient-doctor relationship and patient care if
KEY POINTS

- The majority of the ethnic populations probably explains and understands their illnesses in their traditional medical concepts, rather than Western medical principles.

- Chinese consider both traditional Chinese medicine (TCM) and Western medicine to have strengths and weaknesses. They choose TCM or Western medicine for the specific types of illness.

- They may consider some Western medicines to have significant side effects that make them less compliant with the treatment regimen.

- It is important for medical practitioners in the Western countries to beware of the health concepts of their ethnic patients.

the doctor can show understanding while being proactive in discussing with their Chinese patients of their taking TCM medications. As shown by this study, not only do the Chinese patients take TCM medications for chronic health problems, they may also do so for some acute, though often, self limiting problems. This is different from Westerners who tend to consult complementary practitioners for longstanding conditions for which conventional medicine has not provided a satisfactory solution. ${ }^{13}$ This study also shows that Chinese patients, irrespective of their social class and age, may be taking TCM medications. This is shown by the fact that our participants came from a predominantly lower socioeconomic area with a wide age range. This is again different from the Western populations where users of complementary medicine tend to be in higher socioeconomic groups within the age of 35-60 and have higher levels of education than users of conventional care. ${ }^{13}$

Funding: grant support for this study was received from Conference and Research Grants Committee, the University of Hong Kong.

Conflicts of interest: none.

1 Zollman C, Vickers A. Complementary medicine in conventional practice. BMF 1999;319:901-4

2 Kraut AM. Healers and strangers. Immigrant attitudes toward the physician in America - a relationship in historical perspective. FAMA 1990;263:1807-11

3 Brewer B. An analysis of Hong Kong's health policy. 7 Health Social Policy 1993;4:93-114.

4 Kleinman AM. Depression, somatization and the "new cross-cultural psychiatry”. Soc Sci Med 1977;11:3-10.

5 Kleinman A, Eisenberg L, Good B. Cultural, illness, and care: clinical lessons from anthropologic and cross-cultural research. Ann Intern Med 1978;88:251-8.

6 Chang KC. Food in Chinese culture: anthropological and historical perspectives. New Haven: Yale University Press, 1977.

7 Koo LC. Concepts of disease causation, treatment and prevention among Hong Kong Chinese: diversity and eclecticism. Soc Sci Med 1987;25:405-17.

8 Koo LC. The use of food to treat and prevent disease in Chinese culture. Soc Sci Med 1984;18:757-66.

9 Lau BWK. When and how patients seek medical help? An exploration in Hong Kong. Hong Kong Practitioner exploration in

10 Helman CG. Culture, health and illness. 4th ed. Oxford: Butterworth-Heinemann, 2000

11 Strauss A, Corbin J. Basics of qualitative research. Grounded theory procedures and techniques. London: Sage, 1990.

12 Vickers A, Zollman C. ABC of complementary medicine: herbal medicine. BMf 1999;319:1050-3.

13 Zollman C, Vickers A. ABC of complementary medicine: users and practitioners of complementary medicine. $B M \mathcal{F}$ 1999;319:836-8. 